\title{
High-frequency tinnitus without hearing loss does not mean absence of deafferentation
}

\author{
Nathan Weisz a,b,*, Thomas Hartmann ${ }^{\text {a }}$, Katalin Dohrmann ${ }^{\text {a }}$, \\ Winfried Schlee ${ }^{\mathrm{a}}$, Arnaud Norena ${ }^{\mathrm{c}}$ \\ a Department of Psychology, University of Konstanz, Germany \\ ${ }^{\mathrm{b}}$ INSERM U280, France \\ ${ }^{\mathrm{c}}$ CNRS UMR5020, Université Claude Bernard, France
}

\begin{abstract}
A broad consensus within the neuroscience of tinnitus holds that this audiologic condition is triggered by central deafferentation, mostly due to cochlear damage. The absence of audiometrically detectable hearing loss however poses a challenge to this rather generalizing assumption. The aim of this study was therefore to scrutinize cochlear functioning in a sample of tinnitus subjects audiometrically matched to a normal hearing control group. Two tests were applied: the Threshold Equalizing Noise (TEN) test and a pitch scaling task. To perform well on both tasks relatively normal functioning of inner hair cells is a requirement. In the TEN test the tinnitus group revealed a circumscribed increment of thresholds partially overlapping with the tinnitus spectrum. Abnormal slopes were observed in the pitch scaling task which indicated that tinnitus subjects, when presented with a high-frequency stimulus, relied heavily on input derived from lower-frequency inner hair cells (off-frequency listening). In total both results argue for the presence of a deafferentation also in tinnitus subjects with audiometrically normal thresholds and therefore favour the deafferentation assumption posed by most neuroscientific theories.
\end{abstract}

Keywords: Tinnitus; Tinnitus spectrum; Hearing loss; Dead regions; Pitch scaling

\section{Introduction}

Chronic subjective tinnitus (thereafter termed tinnitus) is a very common audiological symptom (affecting 5-15\% of the population) and is characterized by an auditory perception not concomitant to any physical source. This phantom sound is usually quite simple (e.g., tones, hissing, buzzing, and noise), which therefore distinguishes tinnitus from auditory hallucinations like schizophrenia. Numerous

Abbreviations: DPOAE, distortion product otoacoustic emissions; TEN, Threshold Equalizing Noise; ISI, inter-stimulus-interval; fRef, reference frequency

* Corresponding author. Present address: INSERM Unité 280, 95 Boulevard Pinel, 69500 Bron, France. Tel.: +33 472138916.

E-mail address: weisz@lyon.inserm.fr (N. Weisz). studies have addressed the psychoacoustical properties of tinnitus. Loudness of tinnitus has been reported to be low in the vast majority of cases, just above threshold. Tinnitus has also been characterized in terms of its frequency content. Tinnitus can sound like a noise (buzzing), but interestingly, tinnitus is more often a tonal-like perception (subjects are able to describe their tinnitus on a scale ranging from low to high). The characterization of "tinnitus pitch" is normally achieved by some tone matching procedure (see Henry and Meikle, 2000 for overview). For example, a typical approach would consist in adjusting a sound until the tinnitus subject has the impression that the frequency resembles his/her perceived pitch. Automated procedures using a computer have also been published, in which the subject, e.g., makes higher/lower judgments or gives judgments which of two sounds is closer 
to the tinnitus pitch (Henry et al., 2004). Tinnitus pitch matching has revealed that tinnitus pitch is usually in the high-frequency region. A different approach was suggested by Norena et al. (2002). These authors postulated that tinnitus, while being tonal-like (by opposition with broadband noise), is not strictly tonal and presents a certain bandwith. A procedure has then been developed to describe the "spectrum" of tinnitus. Subjects had to rate $(0-10)$ how much a given tone frequency (among many) contributes to the overall tinnitus perception. This procedure then yields what can be called a "tinnitus spectrum". Generally, the tinnitus spectrum corresponded to the frequency range of hearing loss. This work is in line with the wide-spread notion that tinnitus and hearing loss are related phenomena. Particularly in neuroscientific approaches of tinnitus hearing loss is ascribed an outstanding role as initiating event that triggers neurophysiological processes which are finally perceived as tinnitus sound. Several animal studies have shown that induced hearing loss leads to changes in spontaneous activity, among others generally enhanced activity and more coherent activity in the region of the tonotopic map affected by hearing loss (Norena and Eggermont, 2003). Also changes in tonotopic organization have been reported, with neurons in the deafferented region shifting their characteristic frequency towards frequencies at the edge of the hearing loss (Irvine and Rajan, 1997; Norena et al., 2002). Alterations in spontaneous activity patterns (Weisz et al., 2005b), as well as transient and steady-state neuromagnetic responses (Diesch et al., 2004; Weisz et al., 2005a; Wienbruch et al., in press) have been reported recently in human tinnitus subjects with high-frequency hearing loss. Even though, not quite identical in their research approaches and theoretical assumptions, the importance of hearing loss is not questioned. Thus these approaches will be subsumed under the label 'deafferentation hypothesis' in the following.

On the other hand, it is also known that some tinnitus patients do not show any hearing loss in the audiogram. This result suggests that either hearing loss is not necessary to cause tinnitus or several types of tinnitus exist (hearing loss-induced tinnitus and other types of tinnitus, for instance). Both alternatives pose serious problems for the deafferentation hypothesis. There is however an additional possibility: that the audiogram alone does not always indicate peripheral damage. In other words, the absence of hearing loss does not exclude the possibility of a hearing damage. Attempts to resolve this problem have been made using distortion product otoacoustic emissions (DPOAE) in subjects with normal audiograms. However results have been ambiguous, showing decreases (Shiomi et al., 1997) as well as increases of outer-hair cell activity in the tinnitusfrequency region (Gouveris et al., 2005).

The first goal of the present study was to take a closer look at inner-hair cell damage, in subjects with relatively normal hearing thresholds. For this purpose, we utilized the Threshold Equalizing Noise (TEN) test of Moore et al. (2000). The main feature of this diagnostic screening tool is to present a broad-band noise (at different levels), that is designed in such a way that equal noise power (calibrated in SPL) is contained in all cochlear auditory output filters. This is done to control for off-frequency listening (i.e., performing the task with input from neighbouring undamaged/less damaged inner hair-cells). When the threshold level for a pure tone, which the participant is instructed to detect is much above the noise level, then this is an indicator for the presence of off-frequency listening and a dead region at this frequency. As a rule of thumb the instructions determine $10 \mathrm{~dB}$, although this too has to be seen as rather continuous in reality rather than dichotomous (on-off). As another approach to investigate inner-hair cell damage we decided to do a pitch scaling experiment. In this test (see Methods for details), the task of the subject is to divide a subjective pitch dimension spanned by to anchor points (high and low pure tone) into perceptually equal pitch segments (here 4; i.e., adjusting 3 intermediate pure tone). In tinnitus subjects the upper anchor (high-frequency) was placed into the tinnitus spectrum (assessed with the Norena et al. procedure). It is known (Greenwood, 1997; for the simple cases of pure tones) that the solution to this task largely depends on processes in the inner-ear: equal distances on a perceptual level correspond to equal distances on the basilar membrane independent of which anchors are chosen. If the participants utilizes the anchor points the experimenter intended (by

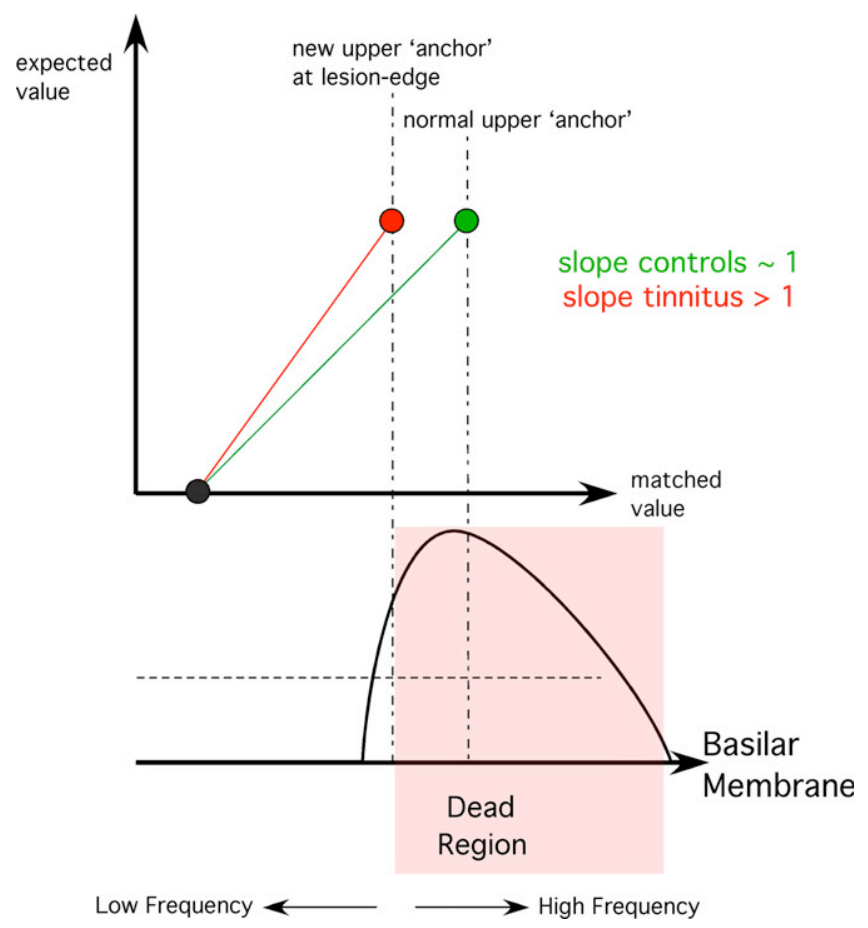

Fig. 1. Outline of the rationale of the pitch scaling study. Assuming that controls have a relatively healthy cochlea a slope of $\sim 1$ (set to be 1 in the graphic) between hypothesized and matched values is expected. If in tinnitus subjects the upper frequency falls into a dead region with severely damaged inner hair cells, then the upper frequency information will be obtained from less damaged lower frequency regions. This should also lead to an altered slope. 
defining the frequency of the pure tone), then when behaviourally matched values are plotted against expected 'ideal' values normally slopes close to 1 should result. According to our rationale of the present study however, sounds presented within the tinnitus spectrum could result in off-frequency listening, i.e., the upper anchor would be shifted. In case of our tinnitus subjects in which highfrequency tinnitus was prevalent, putatively indicating high-frequency hearing receptors damage, a shift towards lower frequencies would be the most likely effect, resulting in slopes larger 1. This idea is based, e.g., on data from psychoacoustical tuning curves, which show that when a signal frequency falls into a dead region, the tip of the tuning curve shifts to low frequencies, indicating that off-frequency listening is taking place (Moore et al., 2000). The rationale is schematically displayed in Fig. 1.

The basic assumption of this study was that if the tinnitus group showed distinctive features in the TEN test and slopes strongly deviating from 1 in the pitch scaling experiment, then the presence of normal audiograms in tinnitus subjects would not disconfirm the deafferention hypothesis.

\section{Methods}

\subsection{Participants}

Eleven tinnitus (Mean $=26.63$ years; $S E=1.90 ; 4$ women) and (for the pitch scaling experiment) 11 controls subjects (Mean $=28.63$ years; $S E=.97 ; 4$ women) who self-reported their audition as normal (e.g., no selfperceived hardness of hearing, no previously diagnosed audiological disorders, e.g., Morbus Meniere etc.) participated in the study. Tinnitus participants all reported of having this condition continuously for more than six months (chronic tinnitus) for which no external sound source could be diagnosed (subjective tinnitus). All subjects were students which were recruited via postings at the University of Konstanz. A summary of tinnitus and audiometry related information can be found in Table 1. Data from controls subjects is also included here (NA indicating that data is not available). It is important to emphasize that overall both groups have hearing thresholds within normal limits (see Fig. 2).

\subsection{Tests and procedure}

Tinnitus subjects underwent following experimental procedure: (1) assessment of tinnitus spectrum, (2) pitch scaling test, and (3) dead regions diagnostic (including pure tone audiometry). For control subjects we assessed pure tone audiograms and pitch scaling data. These tests will be described in the following:

1. Tinnitus spectrum. Conforming to the approach published by (Norena et al. (2002)) pure tones of varying frequencies (duration $=1 \mathrm{~s}$ ) were presented to the subject one at a time. Following frequencies were chosen:
250, 500, 1000, 1500, 2000, 3000, 4000, 5000, 6000, 7000 , and $8000 \mathrm{~Hz}$. Each trial consisted of two parts: at first the subject was requested to adjust the loudness of the tone such that it matched the perceived loudness of the tinnitus. After this she/he had to rate $(0-10)$ how much the tone frequency contributes to her/his tinnitus spectrum. Overall each frequency was presented four times in a pseudorandom order. The first round (i.e., in which all 11 frequencies were presented once) was considered as practice round and was not considered in the analysis. In cases of bilateral tinnitus the procedure was done for both ears. Here, only part of the data will be displayed, which is relevant for our research question. The experiment was run using GUIs implemented under Matlab 5 on Mac OS 9.2.

2. Pitch scaling. The test started off by the subject having to adjust the loudness of 9 tones $(400,550,756,1427,1962$, 2696, 3706, 5093, and $7000 \mathrm{~Hz}$ ) to a fixed $1 \mathrm{kHz}$ reference tone $(80 \mathrm{~dB}$ SPL; duration $=1 \mathrm{~s})$. These values were used to adjust the loudness of all possible frequencies between 400 and $7000 \mathrm{~Hz}$ to approximately equal loudness by means of spline interpolation. In the actual pitch scaling task subjects were instruct to segment a subjective pitch dimension spanned by an upper and lower frequency ('anchors', respectively), into 4 equal parts by regulating the frequency of three intermediate tones. The subject was able to check the appropriateness of the response by playing the entire sequence, which was varied either between low-to-high and high-to-low (tone duration: $1 \mathrm{~s}$; no ISI between tones). The sequence has been shown to have an (opposing) impact on responses, which is cancelled out by changing sequence direction (Greenwood, 1997). The actual experiment consisted of 10 rounds, preceded by four practice trials. Tests were conducted for one ear only, which was chosen either on tinnitus laterality (in unilateral cases) or for which ear a broader tinnitus spectrum was more easily detectible. The low anchor remained constantly $400 \mathrm{~Hz}$. The upper anchor was chosen from frequencies where the tinnitus spectrum reached a plateau, which means that at least 3 frequencies had to be markedly elevated: from these frequencies the second lowest frequency was chosen as upper anchor. This frequency corresponded to frequencies between 6 and $8 \mathrm{kHz}$. Accordingly, upper anchors were set within this frequency region in the most of control subjects too. Five control subjects had an upper anchor of $3 \mathrm{kHz}$. As can be theoretically expected (see introduction; Greenwood, 1997) upper anchor frequency did not have an influence on pitch scaling slope (tested by comparing individual slopes of subjects tested with $3 \mathrm{kHz}$ vs. the rest of the non-tinnitus subjects via $t$-test; $t_{9}=1.12, p=.30$ ) results of these subjects were grouped together. The experiment was run using GUIs implemented under Matlab 5 on Mac OS 9.2.

3. Dead regions. Following the pitch scaling experiment dead region diagnostics were undertaken using a the Threshold Equalizing Noise (TEN) test (Moore et al., 
Table 1

\begin{tabular}{|c|c|c|c|c|c|c|}
\hline ID & Sex & Age & T-side & $\mathrm{P}$-scale ear & F upper anchor & Dead-region? \\
\hline ah & Female & 37 & Right dom. & Right & 7500 & Yes, right \\
\hline co & Female & 31 & Right & Right & 7000 & Yes, right \\
\hline $\mathrm{dg}$ & Female & 22 & Bilateral & Right & 7000 & No \\
\hline $\mathrm{dk}$ & Male & 36 & Right dom. & Right & 6000 & Yes, right \\
\hline ds & Male & 30 & Bilateral & Right & 6000 & Yes, left \\
\hline hk & Male & 22 & Left dom. & Reft & 7000 & Yes, bilateral \\
\hline hl & Female & 19 & Bilateral & Left & 7000 & Yes, right \\
\hline sg & Male & 29 & Right dom. & Right & 7500 & No \\
\hline nn & Male & 24 & Left dom. & Left & 7500 & Yes, left \\
\hline hf & Male & 20 & Right dom. & Right & 8000 & Yes, left \\
\hline se & Male & 23 & Right dom. & Left & 7000 & No \\
\hline at & Female & 29 & NA & Left & 3000 & NA \\
\hline im & Male & 27 & NA & Left & 3000 & NA \\
\hline nw & Male & 29 & NA & Right & 3000 & NA \\
\hline ha & Male & 33 & NA & Right & 7000 & NA \\
\hline pe & Male & 33 & NA & Right & 7000 & NA \\
\hline dd & Female & 27 & NA & Right & 3000 & NA \\
\hline ss & Female & 29 & NA & Right & 3000 & NA \\
\hline bk & Male & 31 & NA & Left & 7000 & NA \\
\hline $\mathrm{ms}$ & Male & 30 & NA & Left & 6000 & NA \\
\hline $\mathrm{cm}$ & Male & 24 & NA & Right & 7000 & NA \\
\hline $\mathrm{mi}$ & Female & 23 & NA & Left & 7000 & NA \\
\hline
\end{tabular}

NA, not available.
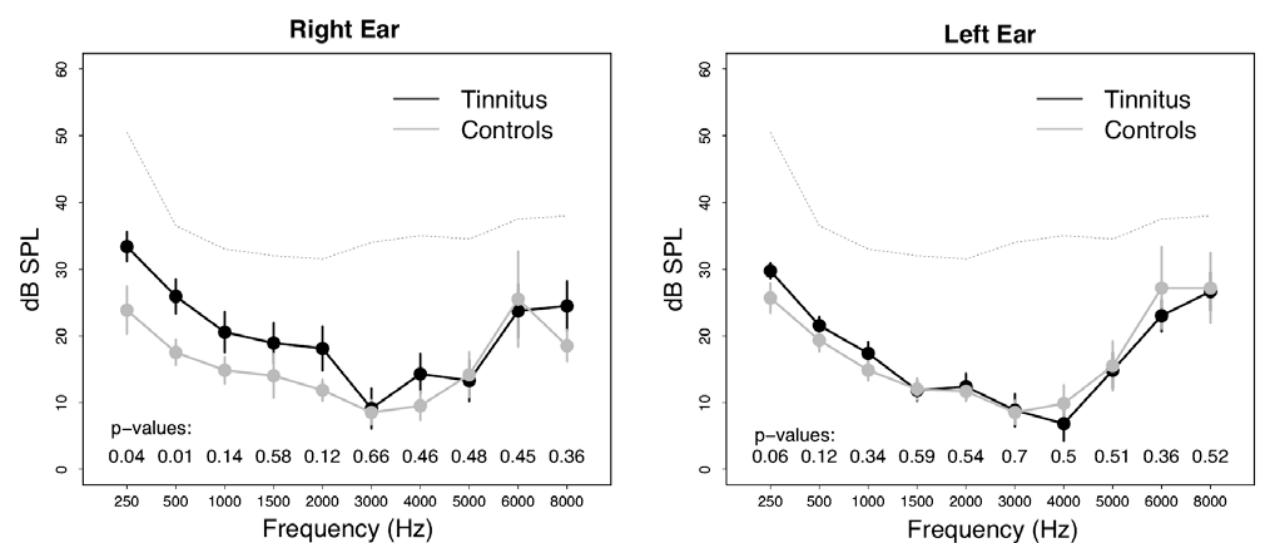

Fig. 2. Comparison of hearing thresholds for the tinnitus and control group. Differences are virtually absent, particularly at higher frequencies ( $>1 \mathrm{kHz})$. The dotted line represent ANSI norms for normal hearing (ANSI S 3.6-1996). Note that according to this criterium both groups exhibit thresholds well within the normal range.

2000). This test was the last in the sequence of test to exclude the bias that could occur when choosing upper anchors for the pitch scaling experiment after knowledge of possible dead regions. In this test sounds are played from a CD (played using Sony CMT-EX1 CD player) which is connected to a clinical audiometer (Interacoustics AC40). All sounds and the noise are calibrated in SPL. The main idea of this test is to account for off-frequency listening which is not detectible from normal clinical audiograms. For each frequency, thresholds are first measured without TEN (pure tone audiometry). Thereafter, TEN is presented with varying degrees of intensity, here: 40,60 , and $80 \mathrm{~dB}$. According to the instruction two conditions should be fulfilled to speak of a dead region at a certain frequency: the threshold should be at least $10 \mathrm{~dB}$ larger than the noise level (more energy needed for off-frequency listening) and the threshold assessed without TEN. Following frequencies were tested: 250, 500, 1000, 1500, 2000, 3000, 4000, 5000,6000 , and $8000 \mathrm{~Hz}$. In order to compare the results of this test with the results of the tinnitus spectrum measurement across subjects, the frequency with the worst result on the $80 \mathrm{~dB}$ noise-level was chosen as reference frequency (fRef).

4. The tinnitus spectrum and the TEN-test threshold was then also averaged for the 3 lower and 3 higher frequencies tested. Since the reference frequencies were usually very high $(\geqslant 6000 \mathrm{~Hz})$ we were rarely able to test all upper 3 frequencies, so that results are displayed for one higher frequency only. 


\subsection{Statistics}

To assess how well groups matched concerning pure tone audiometric thresholds $t$-tests were calculated for each frequency.

In order to be able to average across subjects pitch scaling data were transformed to values between 0 and 4 (the lower anchor being 0 and the upper anchor being 4). The matched values for the intermediate three frequencies (i.e., excluding the fixed anchors) was then entered into a linear mixed effects model using a library implemented in R (Pinheiro and Bates, 2000; R Development Core Team, 2004) and group (tinnitus vs. controls) and 'theoretically correct' frequency (1, 2, and 3) were used as predictors. This approach is similar to a linear regression but besides of population associated fixed effects (here: group and frequency) this model also treats so-called random effects that are associated with the individiual experimental unit (here: subject).

For the three intermediate frequencies, we predicted the pitch scaling slope between theoretically expected and empirically matched values to be higher for the tinnitus group. In our statistic this would be indicated by a significant interaction between the factors group and frequency. Restricted maximum likelihood was used to estimate the coefficients and Compound Symmetry was assumed as correlation structure. The superiority of the inclusion of a ran- dom factor as compared to a standard linear regression approach was validated (Likelihood ratio test: $\chi^{2}=8.60$, $p<.003)$.

\section{Results}

\subsection{Pure tone audiometry}

In Fig. 2, the results of the pure tone audiometry are depicted. Overall both groups exhibited thresholds that were on average well within the range of normal hearing (ANSI S 3.6-1996). At frequencies below $500 \mathrm{~Hz}$ there appears to be slightly increased hearing levels in tinnitus as compared to controls, whereas the higher frequencies are not different.

\subsection{Tinnitus spectrum}

As mentioned above, a region in which spectrum ratings reached a plateau was detected by visual inspection. The result is displayed in Fig. 3( $a$ and b; red line), which shows the typical pattern which can be seen in tinnitus subjects. This usually consists in a rather abrupt change from relatively low ratings to higher values which remain elevated over several frequencies. Ratings from the plateau $(f-1$, fRef, $f+1)$ were significantly higher than the two lower non-plateau frequencies $\left(f-2, f-3 ; t_{10}=4.93, p<.001\right)$.
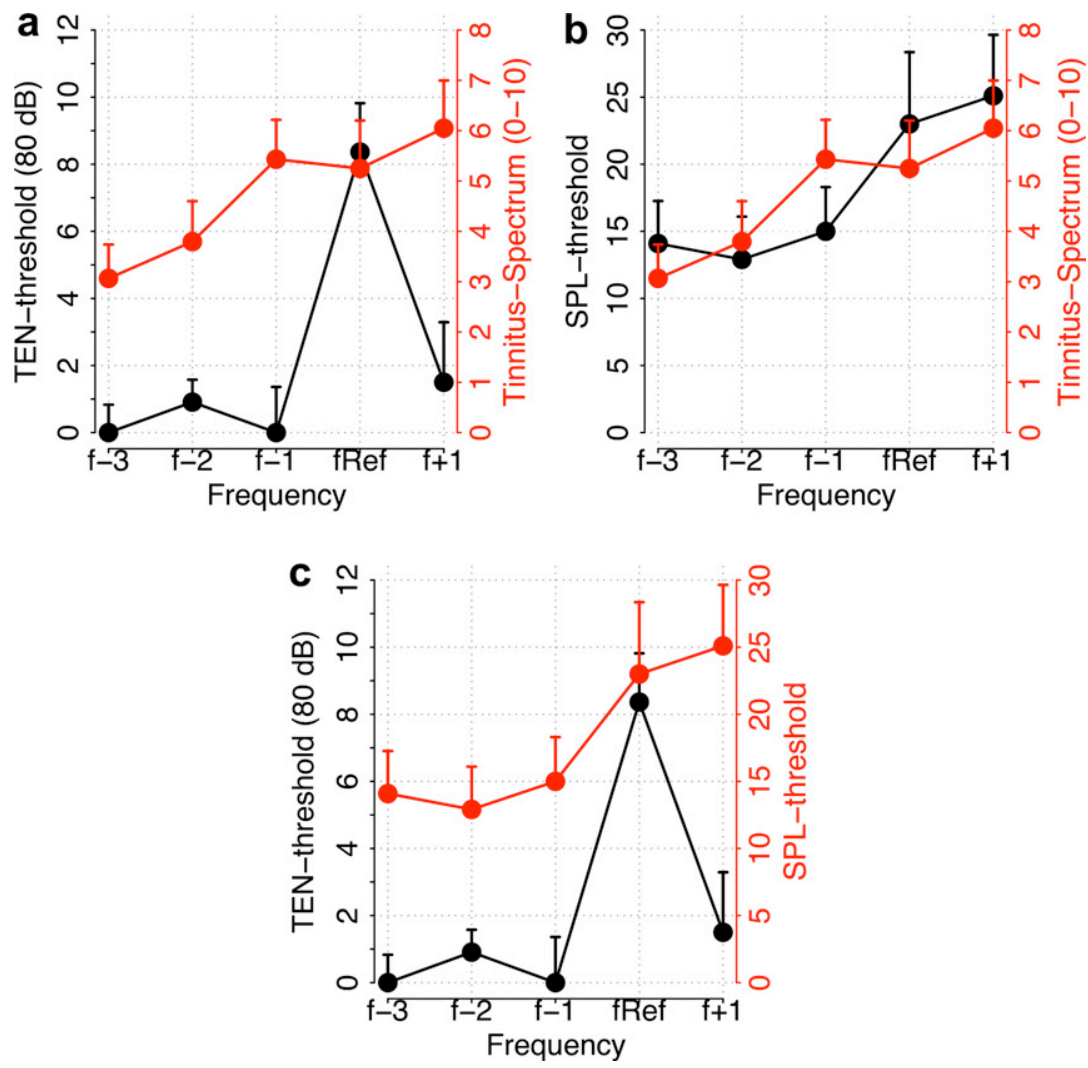

Fig. 3. The overlay (Mean $\pm \mathrm{SE}$ ) of tinnitus spectrum and the TEN test thresholds show that a circumscribed damage exists within the frequency range that is rated as being similar to the tinnitus percept $(\mathrm{A})$. This is the case even though SPL hearing thresholds appear relatively normal ((B) and $(\mathrm{C})$; see also Fig. 2). Meaning of the line colors can be taken from the corresponding axes colors. (For interpretation of the references to colour in this figure legend, the reader is referred to the web version of this article.) 
From this plateau the second lowest frequency was chosen as reference frequency (which was later used as upper anchor in the pitch scaling task).

\subsection{Dead regions}

The thresholds obtained by the TEN test are displayed in Fig. 3( $\mathrm{a}$ and $\mathrm{b}$ ). It can be seen that thresholds are low over a wide range of frequencies $(f 3-1)$ but rises strongly at the reference frequency (fRef) to drop again at the measurement frequency above fRef. Although the average does not exceed $10 \mathrm{~dB}$, it does come very close (Mean: 8.4, $S E=1.5$ ). Eight out of eleven tinnitus participants exhibit thresholds above $10 \mathrm{~dB}$ for the ear in which the pitch scaling procedure was undertaken. The increase in threshold at the reference frequency corresponds to an increase in the SPL calibrated pure tones audiogram (i.e., without noise; Fig. 3c). However TEN-thresholds return almost to baseline values, while the pure tone thresholds remain elevated. Regarding the relation with the tinnitus spectrum that there is an overlap for the two highest frequencies (fRef and $f+1$ ) in the pure tone audiogram (Fig. 3b), but the increase already commences before thresholds rise $(f-1)$. For the TEN-test assessed thresholds this correspondence is only given for the reference frequency.

\subsection{Pitch scaling}

As can be seen in Fig. 4, in which matched values are plotted against expected values the tinnitus group exhibited a greater slope than the control group, which is driven particularly by the high frequency match. This impression is validated by the linear mixed effects model statistics, yield-

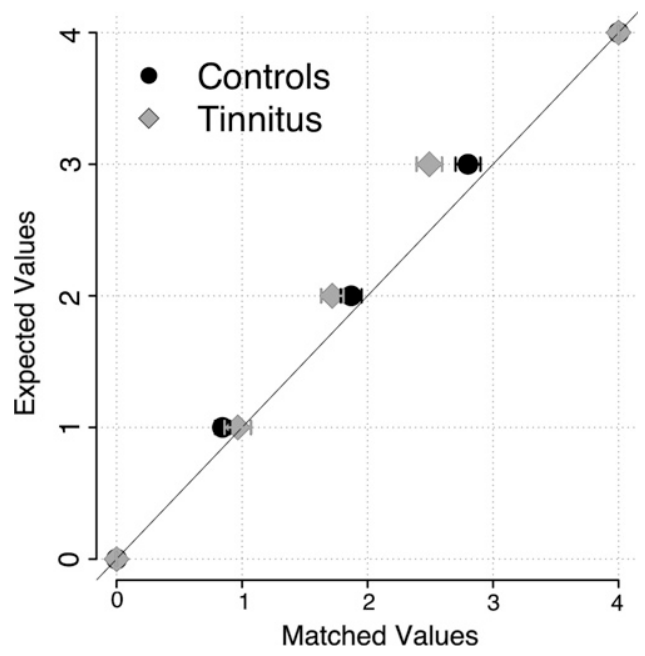

Fig. 4. Depiction of average pitch scaling values $( \pm \mathrm{SE})$ for tinnitus (grey diamonds) and control subjects (black circles). The highest and the lowest points were fixed in the experiment, while the other frequencies could be flexibly adjusted. A difference can be particularly found at high frequencies indicating a shift of processing towards lower frequencies in the tinnitus group. The straight line indicates an ideal match between matched and expected values (slope $=1$ ). ing a significant group difference concerning the slopes $\left(F_{1,42}=6.43, p<.02\right)$.

\section{Discussion}

The main goal of article was to clarify whether a hearing damage is present also in cases where hearing thresholds are relatively normal. This question is of great importance to neuroscientific theory of tinnitus, which holds a deafferentation to be the underlying cause that triggers central reorganization leading to phantom sound perception. In this study we investigated a tinnitus group with normal hearing levels with the so-called TEN test. Furthermore this group was compared with a non-tinnitus group on a pitch scaling task.

Generally, our subjects rated their tinnitus to be of very high pitch, which fits well with previous observations (Vernon and Meikle, 2003). Conforming with the results of Norena et al. (2002) the tinnitus spectrum was not circumscribed but usually changed from low ratings to relatively high ratings and remained elevated. A big difference to the Norena et al. study however was, that in the present study a very young population (students) was investigated, who showed virtually no conspicuities in the pure tone audiogram. Thus it is more difficult to speak of the tinnitus spectrum being an inverse of the audiogram as in the Norena et al. study, in which most subjects suffered severe hearing loss.

From this perspective (i.e., absence of severely elevated thresholds) it is astounding that we could identify dead regions in the majority of subjects $(8 / 11)$. They were on average very circumscribed (i.e., limited to one or two frequencies) and in all cases located within the obtained tinnitus spectrum. This result is a very clear argument against the notion that the absence of audiometric hearing loss (as assessed with standard audiograms) indicates that hearing damage is not a requirement for tinnitus. Although our data show that a dead region in a strict sense (TEN thresholds $>10 \mathrm{~dB}$ ) is not a necessary requirement, it is likely that a strong damage of inner hair cells (and therefore a 'real' deafferentation) is involved in tinnitus. It should be kept in mind that the $10 \mathrm{~dB}$ criterium of the TEN test is a rule of thumb, and does not imply binary conditions (dead, not dead).

According to the notion of off-frequency listening when stimuli are presented in a region in which inner-hair cells are severely damaged, then information from neighbouring less or undamaged regions are used. An above threshold sound (e.g., noise or tone) will not only lead to output from a single auditory filter but also from neighboring ones, the amount depending on the sound level (Moore, 2003). However, this process is not symmetric due to the increasing bandwidth of auditory filters with frequency, leading to greater outputs at higher frequency filters (Moore and Glasberg, 1983). In case of noise induced hearing damage e.g., this asymmetric spread of excitation leads to greater damage at higher frequencies and a relative sparing of 
lower frequencies. As the tinnitus participants exhibited high-frequency tinnitus likely also indicating underlying cochlear dysfunctions in these frequency regions (Norena et al., 2002), it is reasonable to assume for these cases that the hearing system will rely more heavily on lower frequency information. It was thus our hypothesis that this should be made visible in a pitch scaling task, by steeper slopes for the tinnitus group. This would indicate that the subjective upper 'anchor' is not equivalent to the physical one, but is shifted towards lower frequencies. Our result confirms this notion. The fact that some control subjects were tested with $3 \mathrm{kHz}$ as upper anchor does not diminish the validity of the data, since equal perceptual differences in pitch in this task should be reflected in equal distances on the basilar membrane independent of the anchors. This was also the case for our control subjects, in which we found no difference in slope whether the upper anchor was $3 \mathrm{kHz}$ or higher. Taken all our results together, we can make following conclusions:

- Tinnitus is accompanied by severe inner-hair cell damage which can be very circumscribed. This indicates that similar to phantom limb pain a complete deafferentation of certain regions of the auditory cortex is a necessary requirement for developing tinnitus.

- Since this damage overlaps with the assessed tinnitus frequency region, this implies that the tinnitus spectrum only partly reflects the 'real' perceived pitch. The tinnitus spectrum could therefore be a very useful and time economic measure, that reflects areas of cochlear damage that are sufficiently impaired to trigger off development of tinnitus even in subjects with little or no audiometric hearing loss.

- The lesion-edge is the (relatively) undamaged region next to that of severe damage. In the present study the lesionedges would be $f-1$ and $f+1$. Because of the spread of excitation low to high frequencies, we assume however that the low frequency lesion edge is the more important one. Previous studies (Dietrich et al., 2001; Weisz et al., 2005a) have used normal clinical audiograms to define a lesion-edge (always determined as the audiometric edge). This might prove to be insufficient.

- We propose that (in most cases with typical high frequency hearing loss) the predominant tinnitus pitch will be close to the lower lesion-edge frequency. On a neuronal level, this corresponds to the fact that following deafferentation, deprived neurons become responsive to lesion-edge frequencies. Due to off-frequency listening and a stronger allocation of attention towards cues from undamaged regions this pattern is strengthened in the long run: i.e., peripheral damage does not only trigger reorganization on a short time scale, but leads to a remodeling of auditory cortical areas in a usedependent fashion. The result is a large auditory cortical area showing strongly synchronized activity which is tuned especially to the lower frequency lesion-edge.

\section{Acknowledgements}

This study was supported by a grant of the Deutsche Forschungsgemeinschaft (El 101/20). We thank ProAkustic for supplying a clinical audiometer and Anke Trefz for support during data collection.

\section{References}

Diesch, E., Struve, M., Rupp, A., Ritter, S., Hulse, M., Flor, H., 2004. Enhancement of steady-state auditory evoked magnetic fields in tinnitus. Eur. J. Neurosci. 19, 1093-1104.

Dietrich, V., Nieschalk, M., Stoll, W., Rajan, R., Pantev, C., 2001. Cortical reorganization in patients with high frequency cochlear hearing loss. Hear. Res. 158, 95-101.

Gouveris, H., Maurer, J., Mann, W., 2005. DPOAE-grams in patients with acute tonal tinnitus. Otolaryngol. Head Neck Surg. 132, $550-553$.

Greenwood, D.D., 1997. The Mel Scale's disqualifying bias and a consistency of pitch-difference equisections in 1956 with equal cochlear distances and equal frequency ratios. Hear. Res. 103, 199-224.

Henry, J.A., Meikle, M.B., 2000. Psychoacoustic measures of tinnitus. J. Am. Acad. Audiol. 11, 138-155.

Henry, J.A., Rheinsburg, B., Ellingson, R.M., 2004. Computer-automated tinnitus assessment using patient control of stimulus parameters. J. Rehabil. Res. Dev. 41, 871-888.

Irvine, D.R., Rajan, R., 1997. Injury-induced reorganization of frequency maps in adult auditory cortex: the role of unmasking of normallyinhibited inputs. Acta Otolaryngol. Suppl. 532, 39-45.

Moore, B.C., 2003. An Introduction to the Psychology of Hearing. Academic Press, Amsterdam.

Moore, B.C., Glasberg, B.R., 1983. Suggested formulae for calculating auditory-filter bandwidths and excitation patterns. J. Acoust. Soc. Am. 74, 750-753.

Moore, B.C., Huss, M., Vickers, D.A., Glasberg, B.R., Alcantara, J.I., 2000. A test for the diagnosis of dead regions in the cochlea. Br. J. Audiol. 4, 205-224.

Norena, A., Micheyl, C., Chery-Croze, S., Collet, L., 2002. Psychoacoustic characterization of the tinnitus spectrum: implications for the underlying mechanisms of tinnitus. Audiol. Neurootol. 7, 358369 .

Norena, A.J., Eggermont, J.J., 2003. Changes in spontaneous neural activity immediately after an acoustic trauma: implications for neural correlates of tinnitus. Hear. Res. 183, 137-153.

Pinheiro, J.C., Bates, D.M., 2000. Mixed-Effects Models in S and S-Plus. Springer, New York.

R Development Core Team. 2004. R: a language and environment for statistical computing. [Online]. Available by $\mathrm{R}$ Foundation for Statistical Computing http://www.r-project.org.

Shiomi, Y., Tsuji, J., Naito, Y., Fujiki, N., Yamamoto, N., 1997. Characteristics of DPOAE audiogram in tinnitus patients. Hear. Res. 108, 83-88.

Vernon, J.A., Meikle, M.B., 2003. Tinnitus: clinical measurement. Otolaryngol. Clin. North Am. 36, 293-305, vi.

Weisz, N., Wienbruch, C., Dohrmann, K., Elbert, T., 2005a. Neuromagnetic indicators of auditory cortical reorganization of tinnitus. Brain 128, 2722-2731.

Weisz, N., Moratti, S., Meinzer, M., Dohrmann, K., Elbert, T., 2005b. Tinnitus perception and distress is related to abnormal spontaneous brain activity as measured by magnetoencephalography. PLoS Med. 2, e153.

Wienbruch, C., Paul, I., Weisz, N., Elbert, T., Roberts, L.E., 2006. Frequency organization of the $40-\mathrm{Hz}$ auditory steady-state response in normal hearing and in tinnitus. NeuroImage 33, 180-194. 